\title{
Sim
}

\section{Youth Awards}

SRM Annual Meeting, Reno, Nevada, February 14, 2007

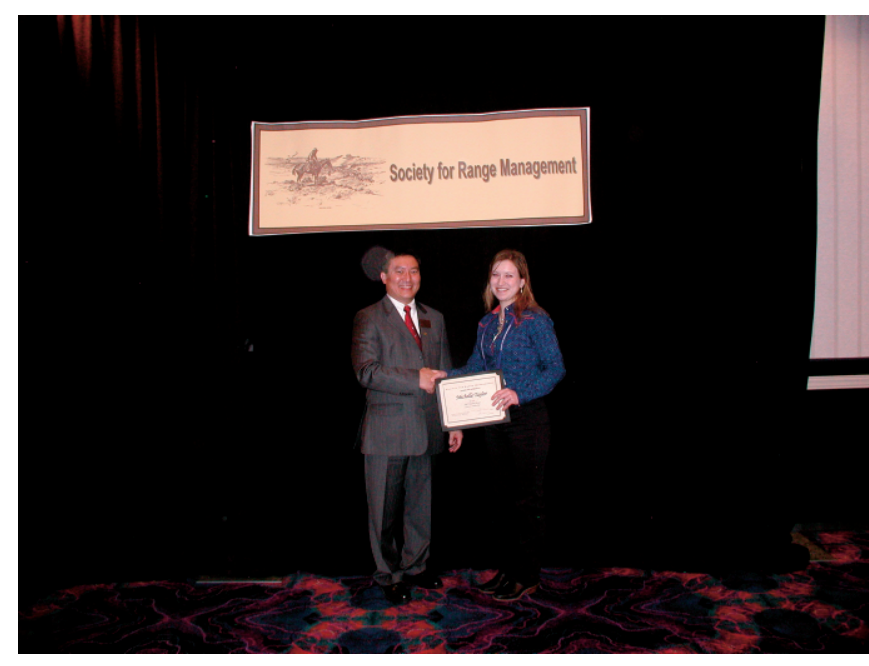

Masonic Range Scholarship: Michelle Taylor, South Dakota State University.

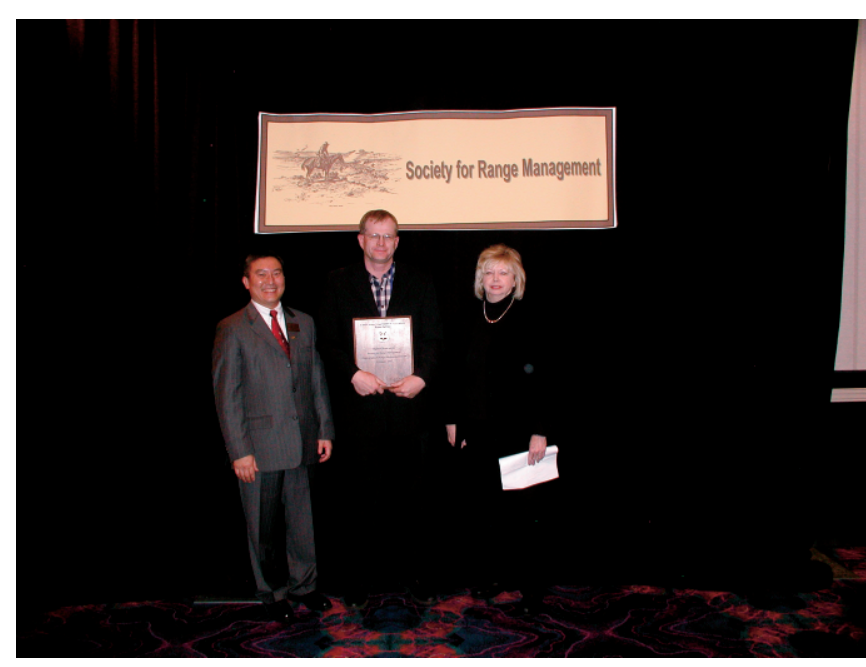

Undergraduate Range Management Exam (UMRE)-Individual: 1stTanner Pollack, University of Alberta with Carpenter Janette Kaiser, USDA Forest Service.

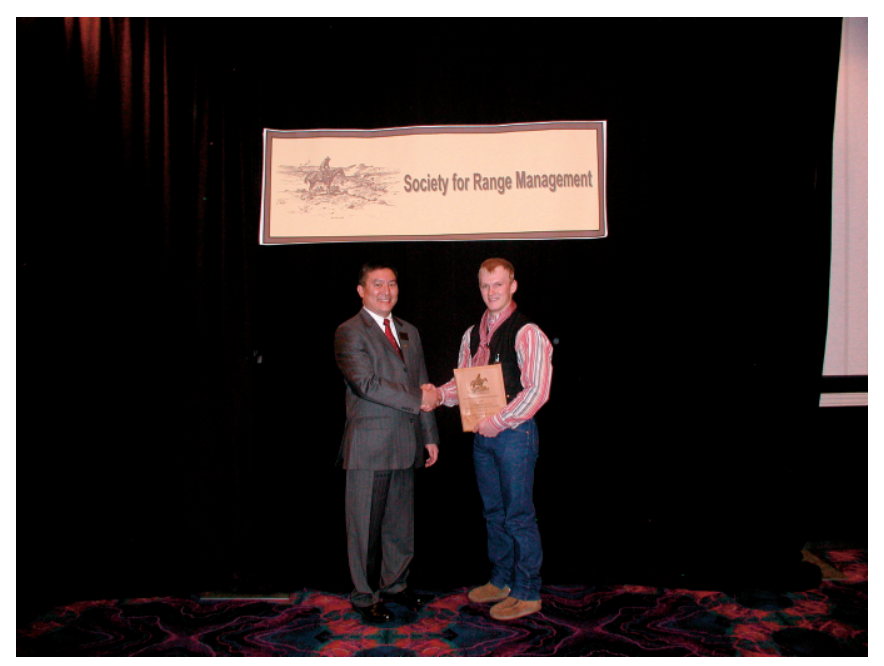

UMRE-Individual: 2nd-Merritt Carpenter, University of Wyoming.

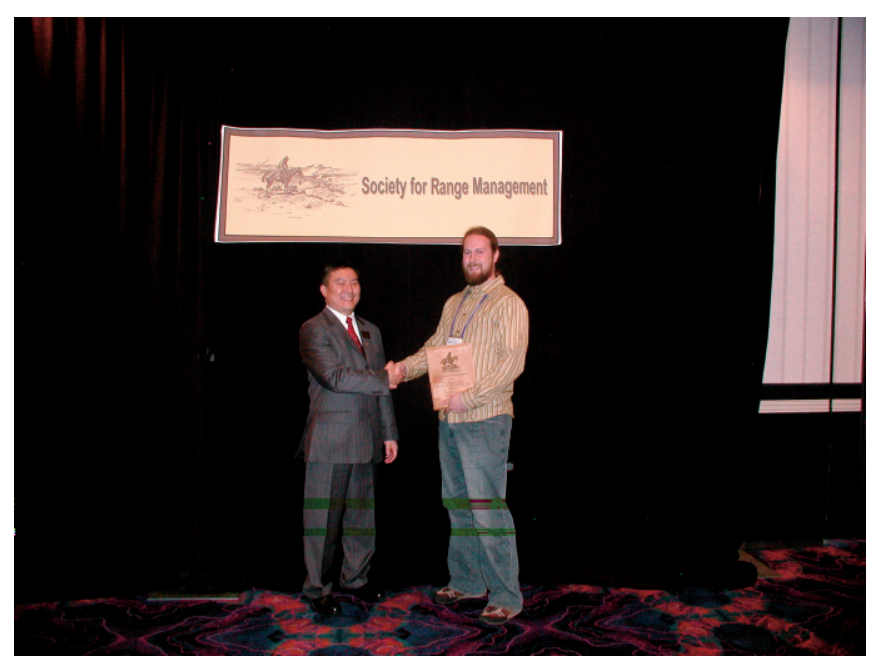

UMRE-Individual: 3rd-Tim Hoelzle, Colorado State University.

Note: Award recipients are pictured with outgoing SRM President John Tanaka. Names in group photos do not reflect the order of those pictured. 


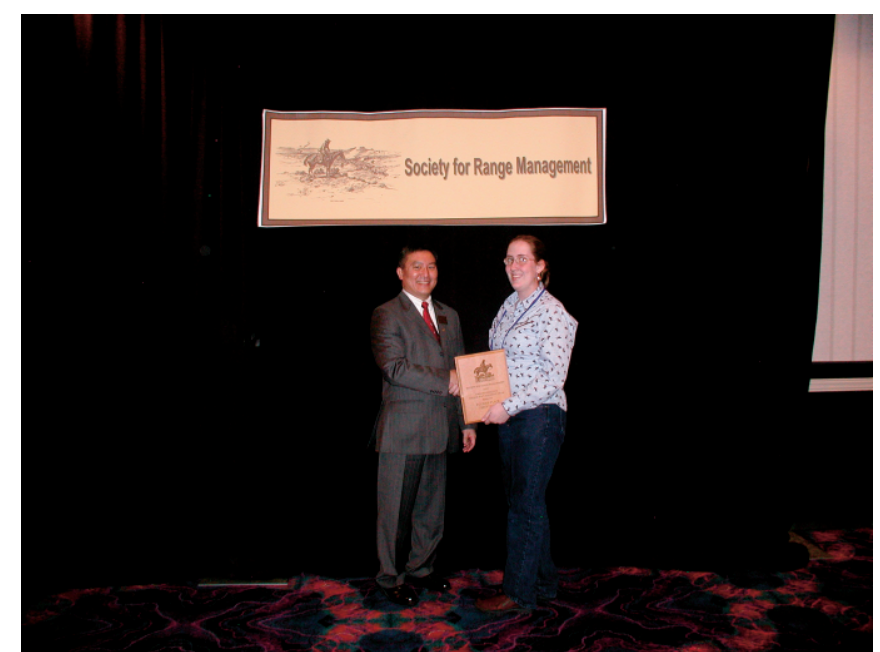

UMRE-Individual: 4th-Sarah Hanlon, University of Wyoming.

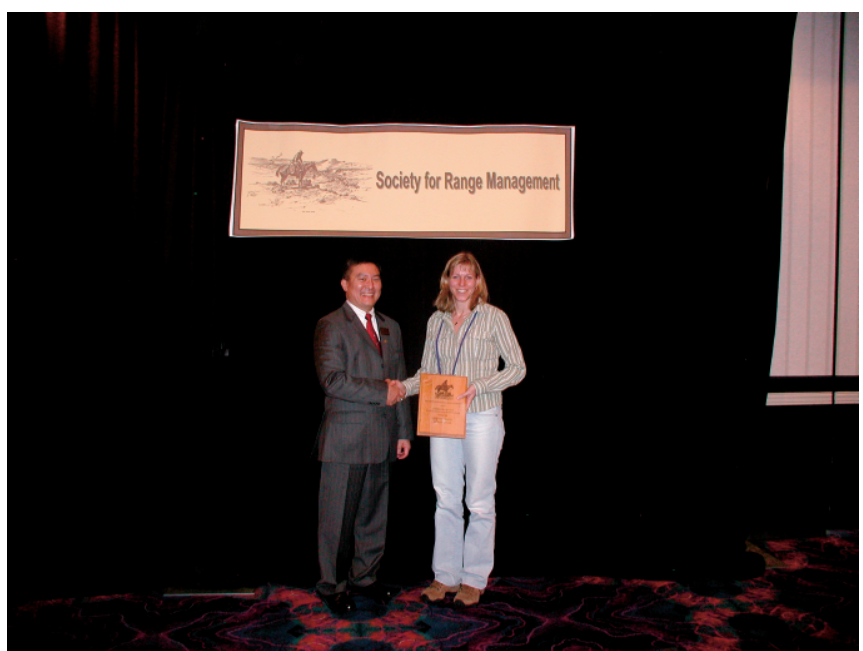

UMRE-Individual: 5th-Amber Greenall, Thompson Rivers University.

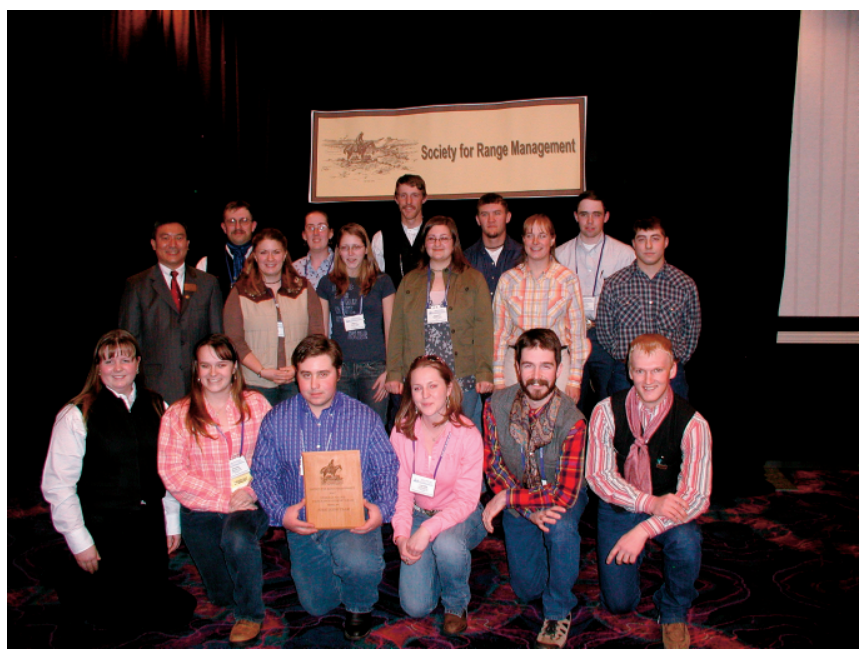

UMRE-Team: 1st-University of Wyoming: Morgan Wolvington, Michelle Auyer, Abigail Martin, Merritt Carpenter, Rollin Winter, Lucas Line, Meghan Reedy, Concetta Brown, Lance Nixon, Sarah Hanlon, Adam Heinle, Elizabeth Parrish, Mae Peterson, Dusty Smith, Josh Jeanneret, Rives White.

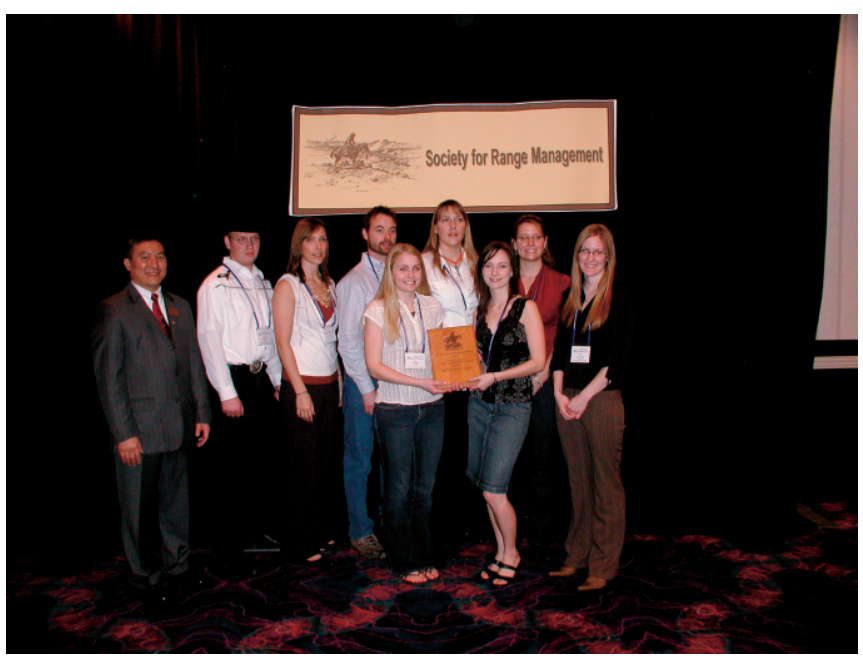

UMRE-Team: 2nd-University of Alberta: Leah Rigney, Darin Sherritt, Brenda Shaughnessy, Erin Schnick, Tisa Bevan, Tianna Magis, Victoria Heidt, Travis Lundberg.

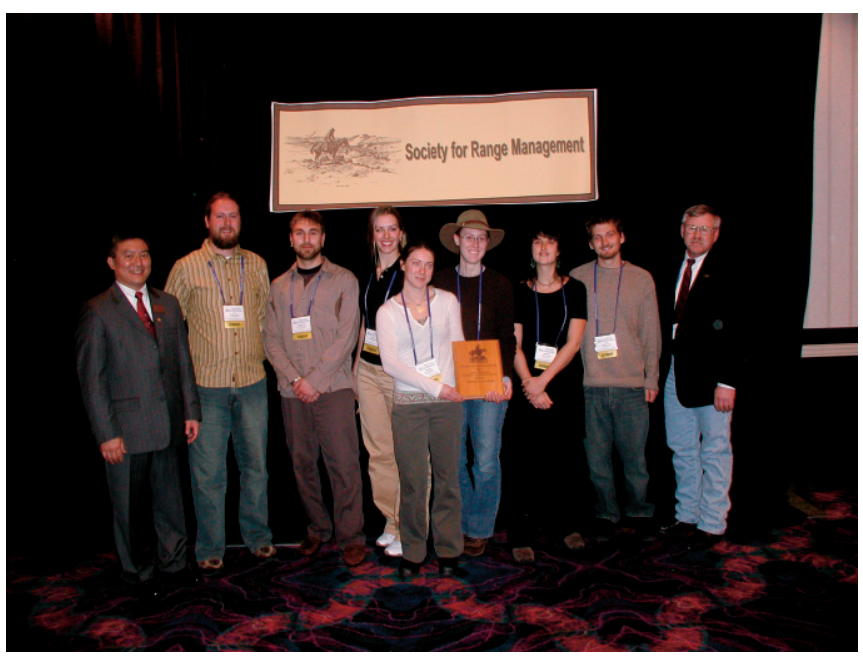

UMRE-Team: 3rd-Colorado State University: Coach Roy Roath, Hannah Varani, Tim Hoelzle, Wendy McBride, Heather Messick. Chris Herron, William Vieth, Maureen Underhill.

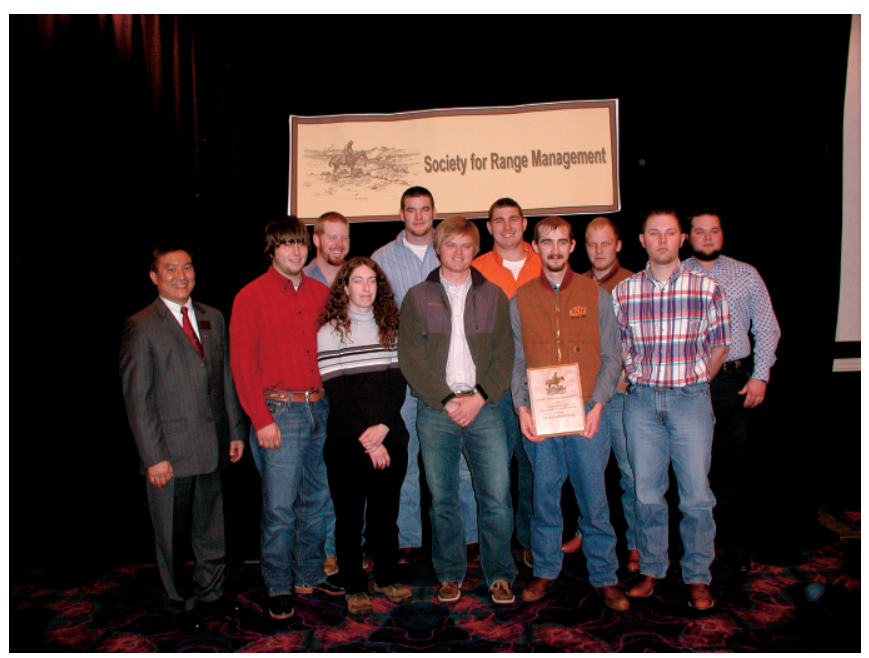

UMRE-Team: 4th-Oklahoma State University: Lauren Wilkerson, Derek Matz, Adam Gourley, Kevin Parsons, Justin Rader, Colin Walden, Rusty Norrie, P. J. Martin, Kevin Spears, Chris Hobbs. 


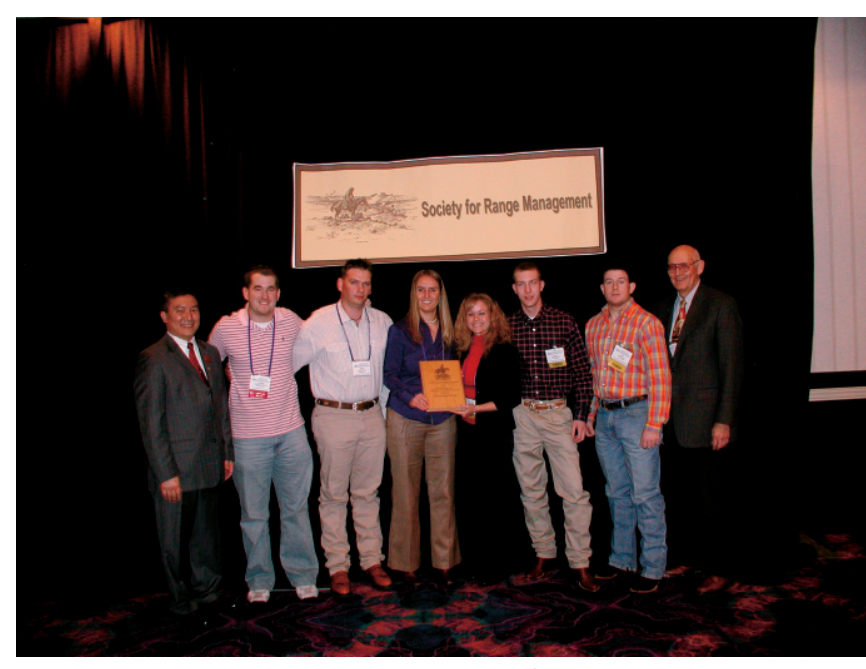

UMRE-Team: 5th-Texas A\&M University: Coach Mort Kothmann, Kimberly Haile, Courtney Ratheal, Rixey Jenkins, Heath Starns, Tyler James, Jonathan Johnston.

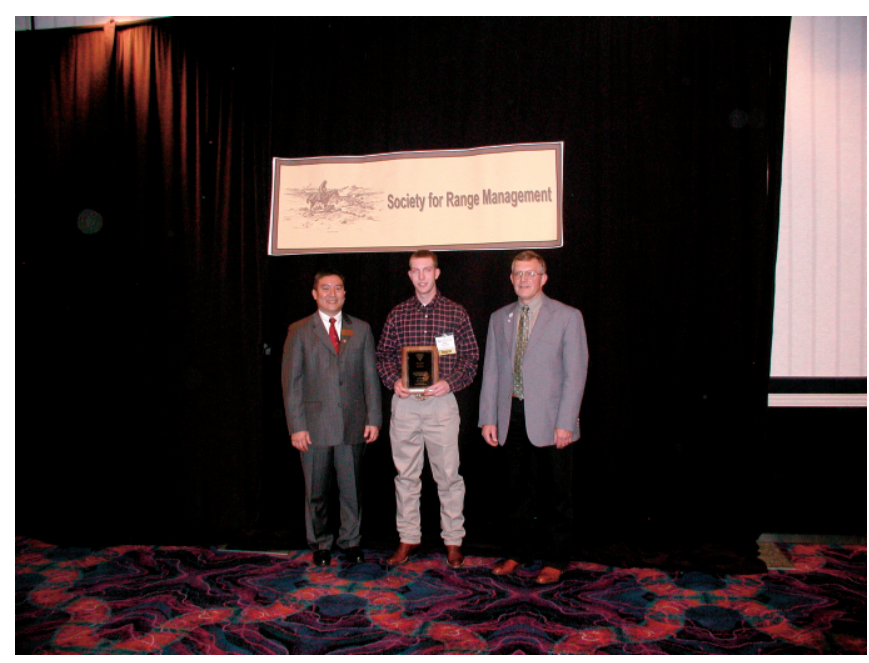

Plant Identification-Individual: 1st-Rixey Jenkins, Texas A\&M University with Bob Bolton, Bureau of Land Management.

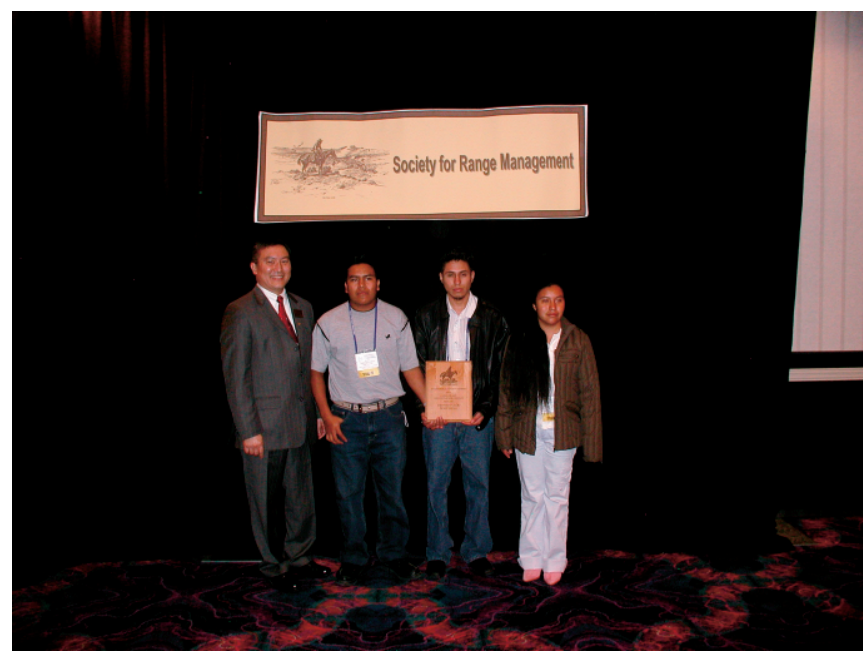

Plant Identification-Individual: 2nd-Tie: Rufino Sandoval Garcia, Universidad de Antonio Narro; Reyna Rojas Garcia, Universidad de Antonio Narro; Ademir Torres Meza, Universidad de Antonio Narro.

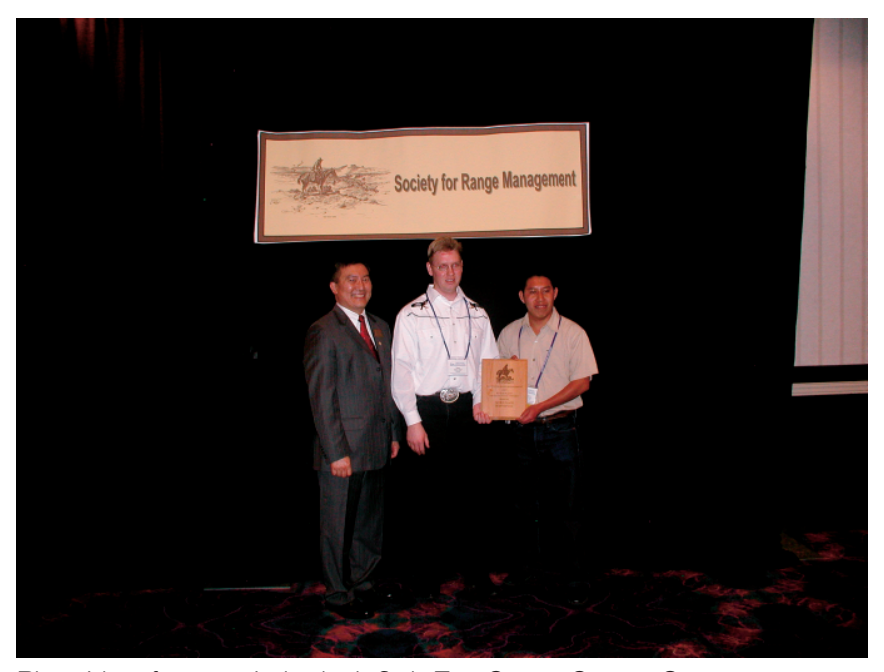

Plant Identification-Individual: 3rd-Tie: Oseas Gomez Cruz, Universidad de Antonio Narro; Darin Sherritt, University of Alberta.

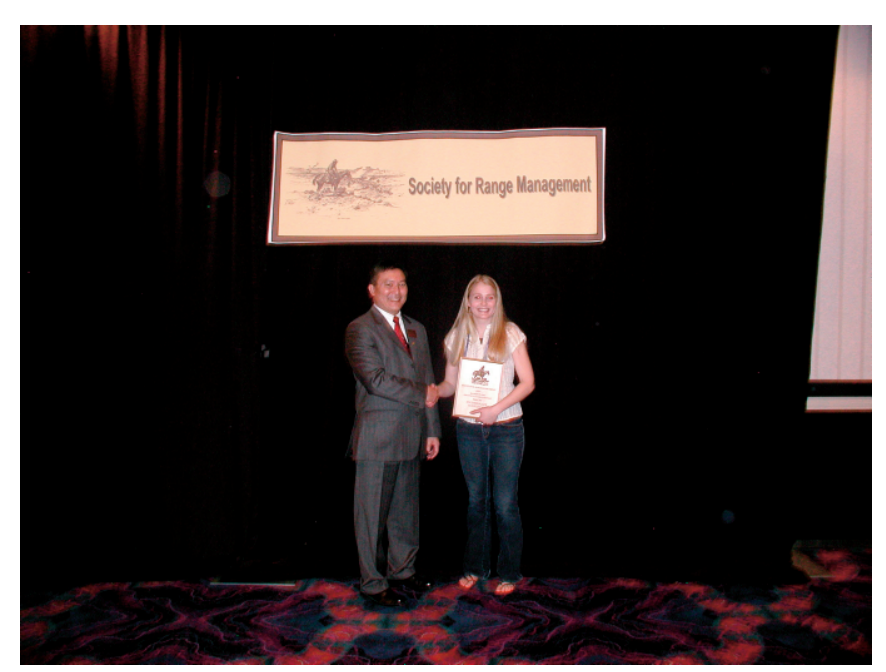

Plant Identification-Individual: 4th-Leah Rigney, University of Alberta.

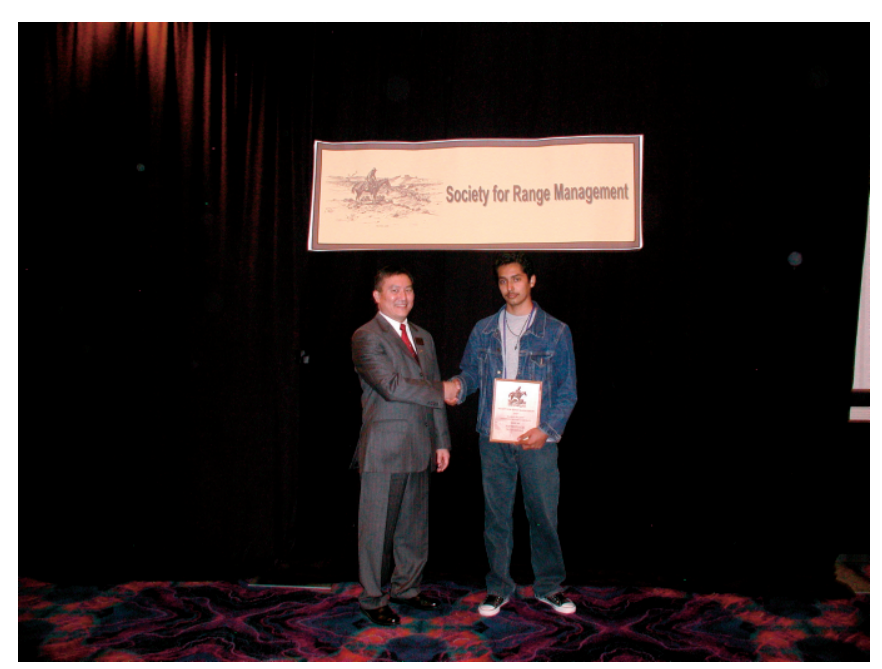

Plant Identification-Individual: 5th-Jose Luis Garcia Franco,

Universidad de Antonio Narro. 


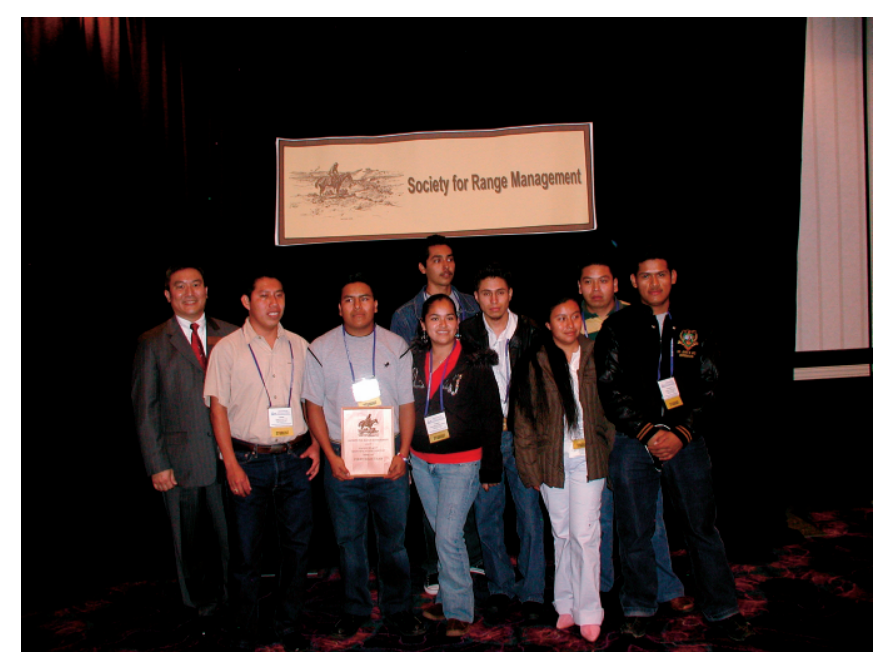

Plant Identification-Team: 1st-Universidad de Antonio Narro: Rufino Sandoval Garcia, Oseas Gomez Cruz, Yuliana Monterrosas Garcia, Reyna Rojas Garcia, Humberto Garcia, Angel Ademir Torres Meza, Victor Hugo Villa Perez, Jose Luis Garcia Franco.

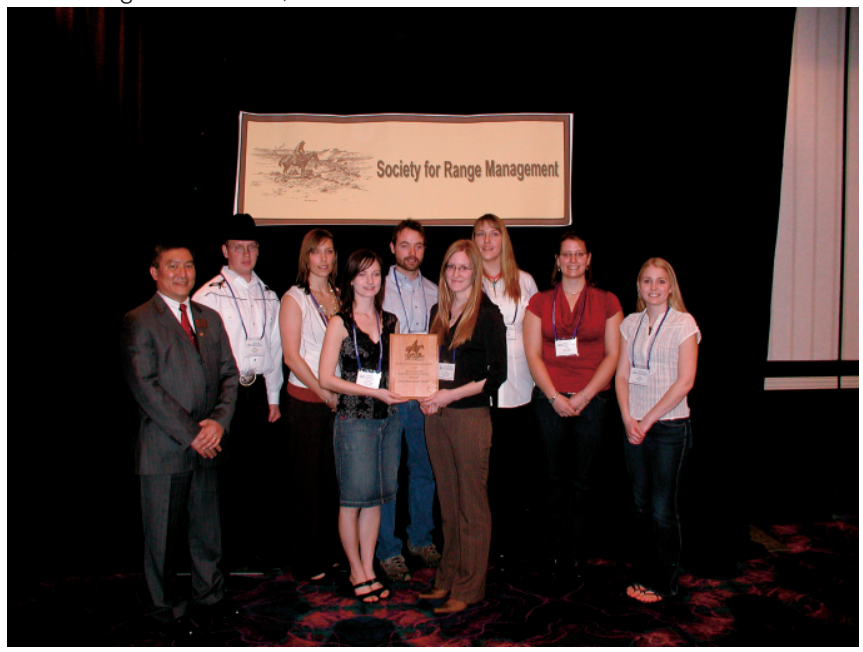

Plant Identification-Team: 2nd-University of Alberta: Brenda

Shaughnessy, Tianna Magis, Darin Sherritt, Victoria Heidt, Erin Schnick, Travis Lundberg, Leah Rigney, Tisa Bevan.

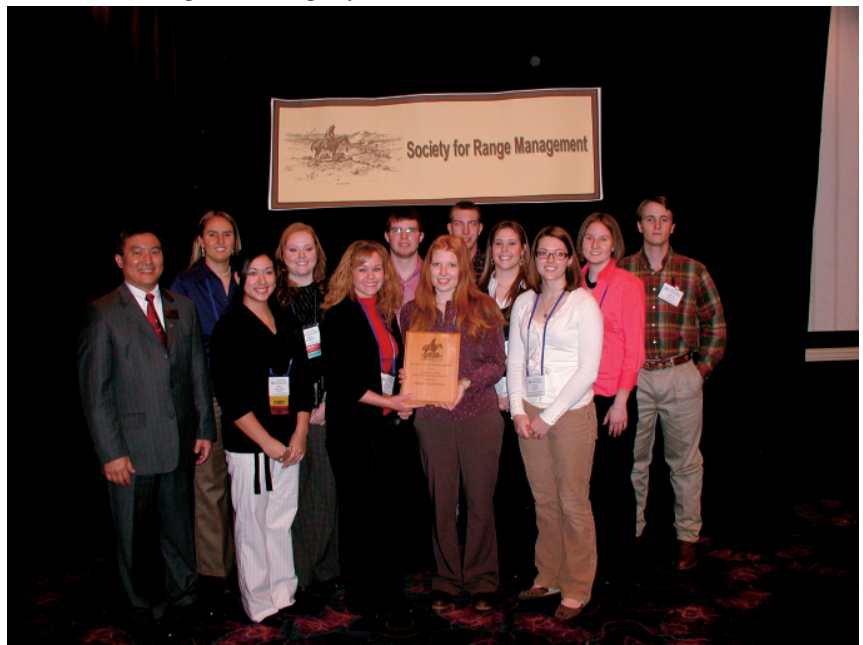

Plant Identification-Team: 3rd-Texas A\&M University: Kimberly Haile, Mindy Gallardo, Ashley Mock, Rixey Jenkins, Courtney Ratheal, Andy James, Mia A. McCraw, Kristen Nelson, Kelly Haile, Katy Weber, Steven Goertz.

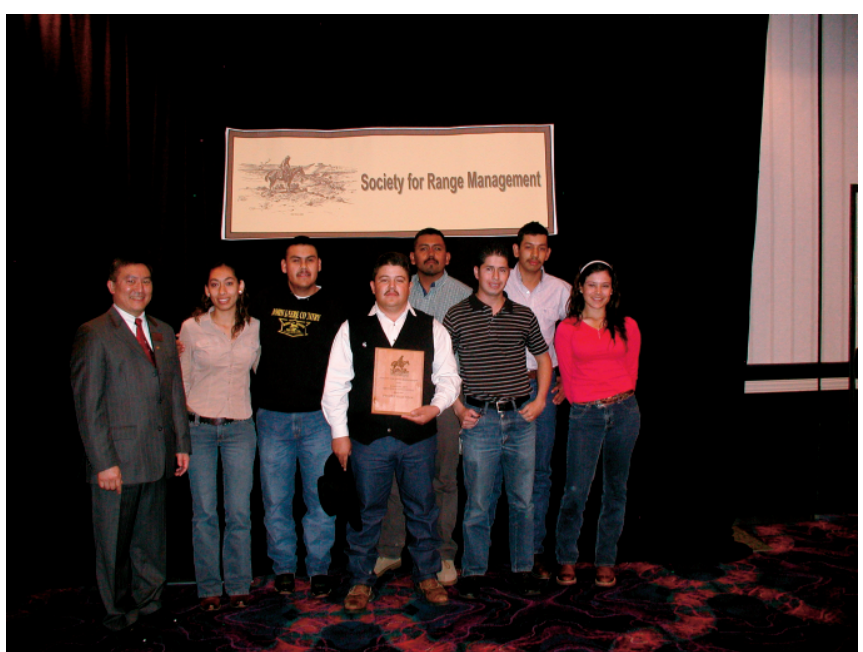

Plant Identification-Team: 4th-Universidad de Chihuahua: Anabel Aguirre Prieto, Giovanni Munoz Romero, Ramon Sandoval Reyes, Jesus Raul Gutierrez-Cruz, Erbey Cormona Ruiz, Manuel Alfonso Moreno Villa.

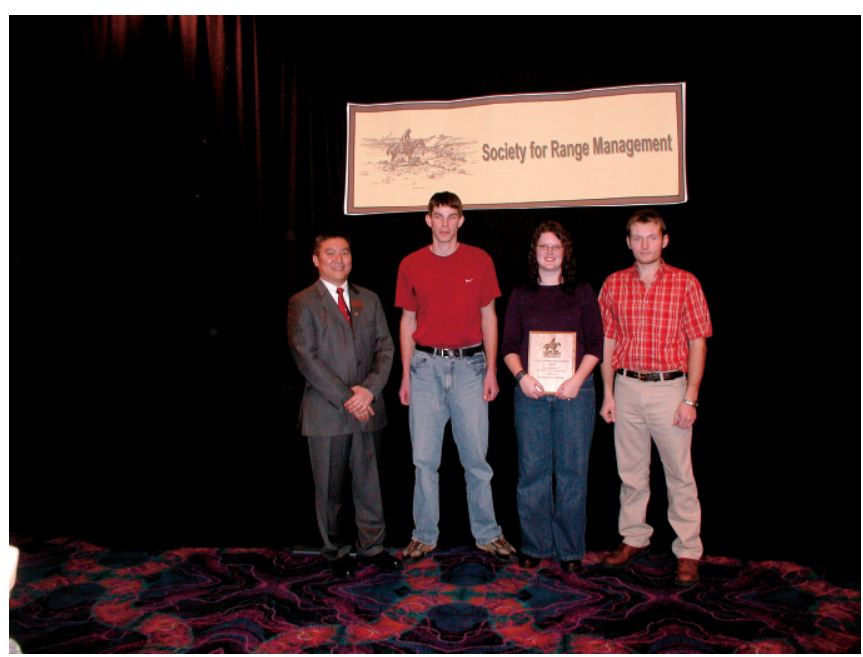

Plant Identification-Team: 5th-South Dakota State University: Coach Gary Larson, Melissa Staples, Jason Biel, Chris Misar.

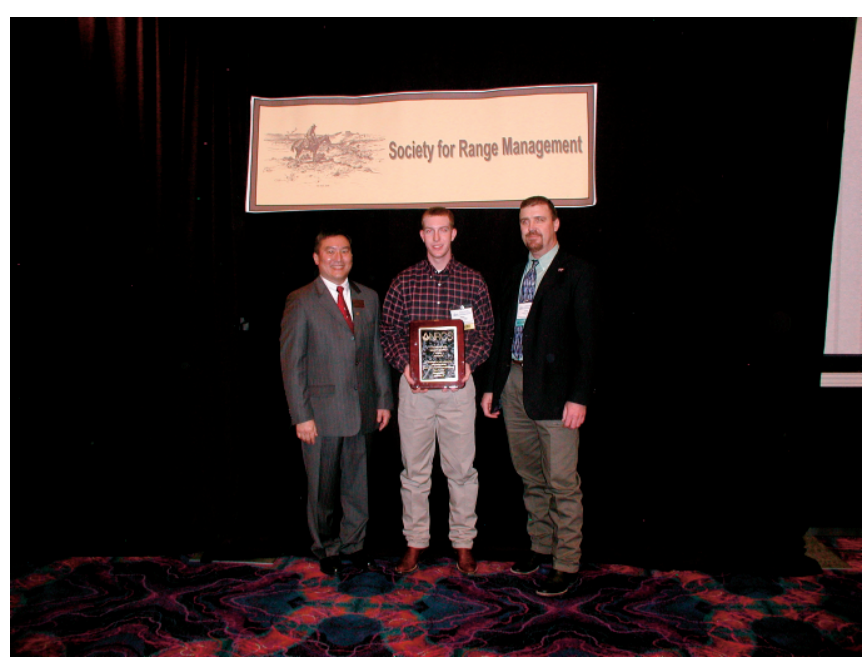

High Combined-Individual: 1st-Rixey Jenkins, Texas A\&M University with Chuck Stanley, USDA Natural Resources Conservation Service. 


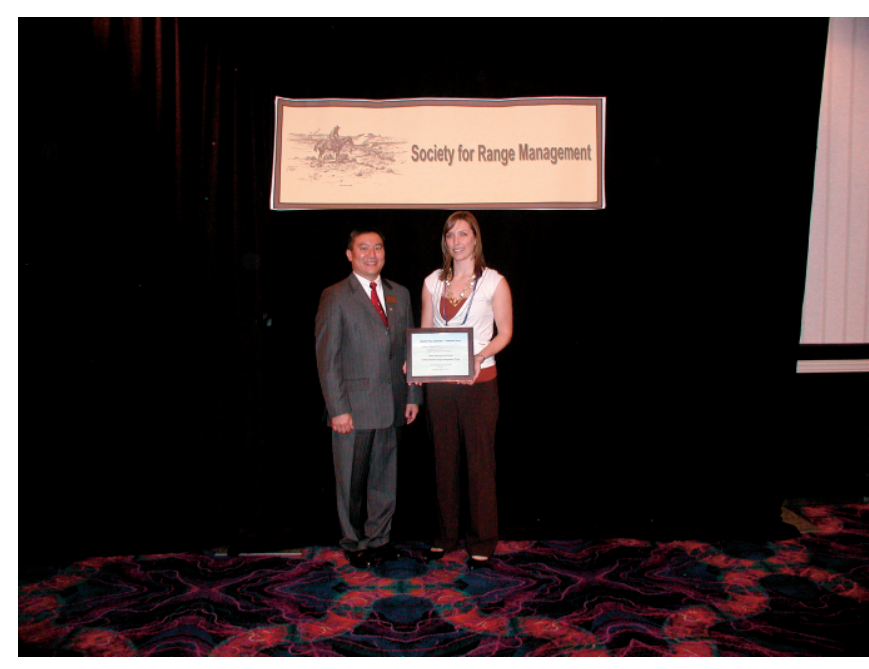

High Combined-Individual: 2nd-Brenda Shaughnessy, University of Alberta.

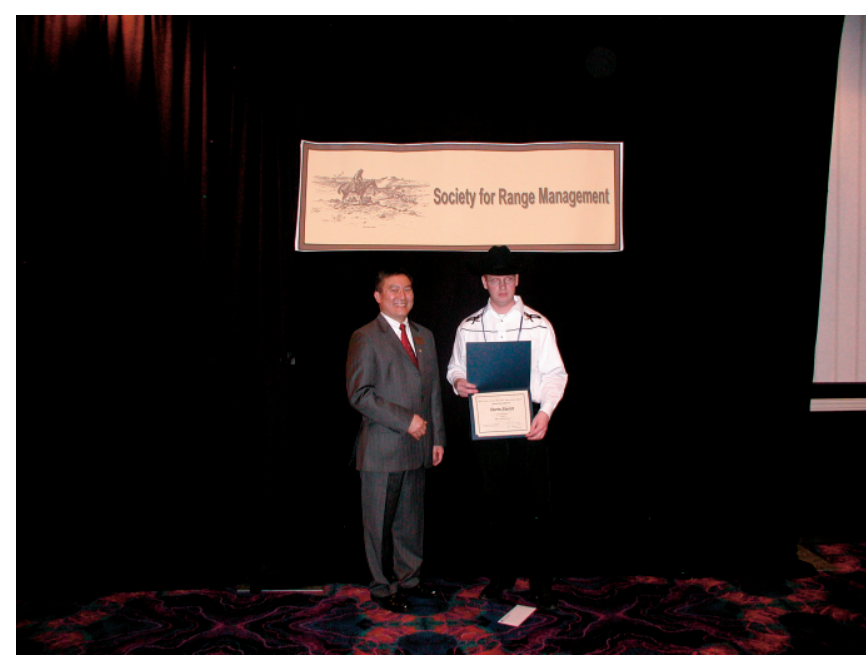

High Combined-Individual: 3rd-Darin Sherritt, University of Alberta.

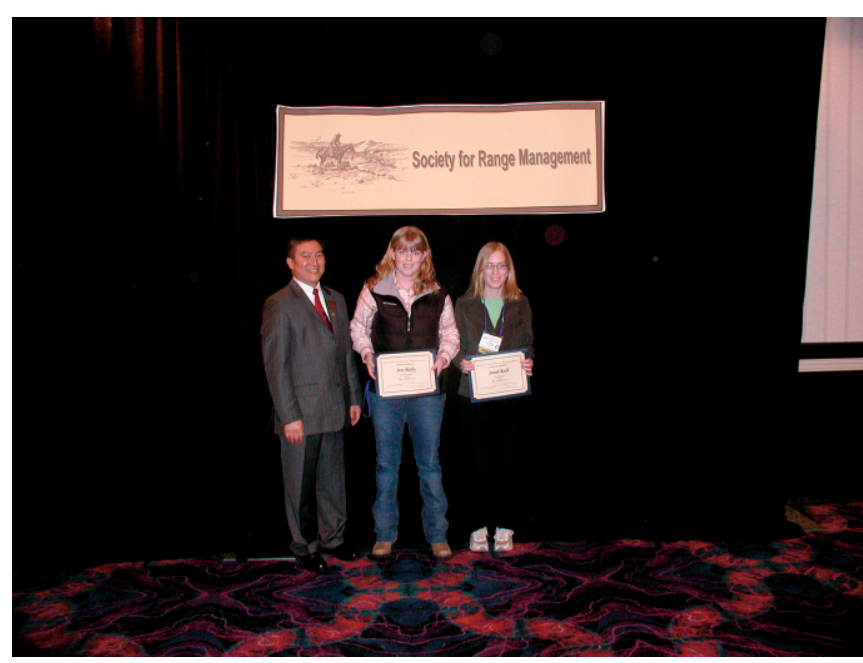

High Combined-Individual: 4th-Tie: Sarah Redd, Utah State University; Jessica Milby, University of Nebraska.

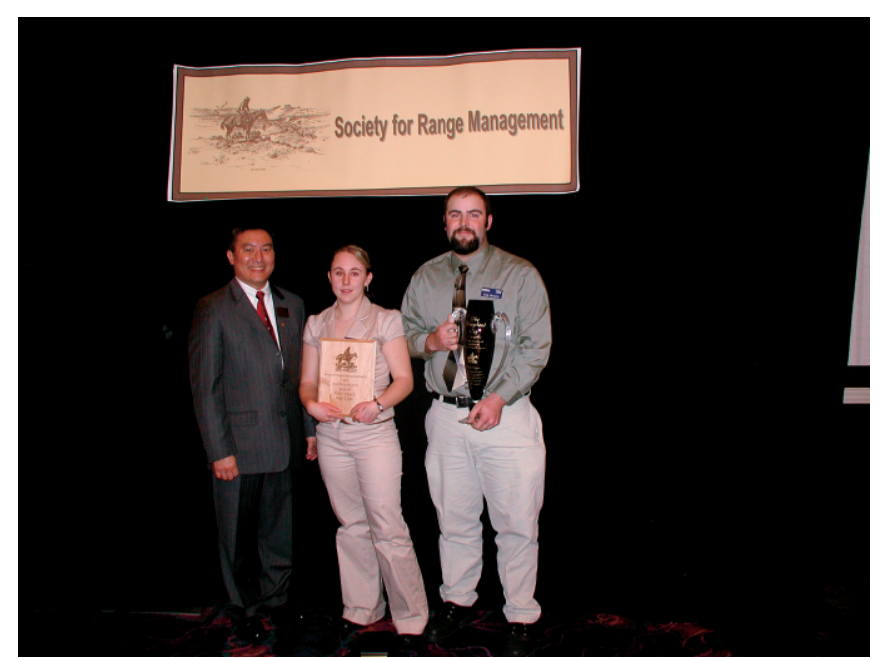

Rangelands Cup-Team: 1st-Utah State University: Kody Menghini, Katie Santini.

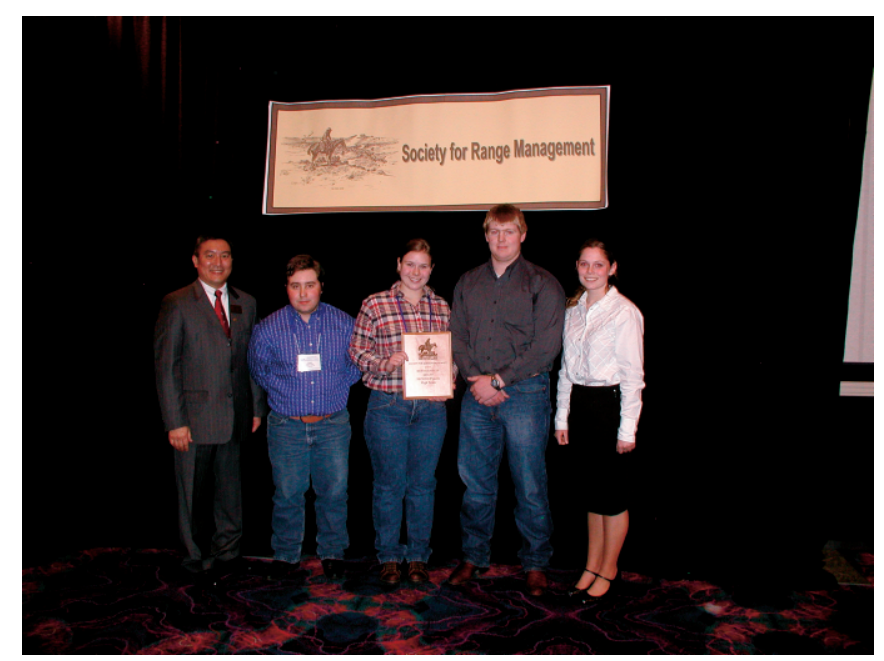

Rangelands Cup-Team: 2nd-University of Wyoming: Alison Iroz, Ashley Whitman, Adam Heinle, Cole Lambert.

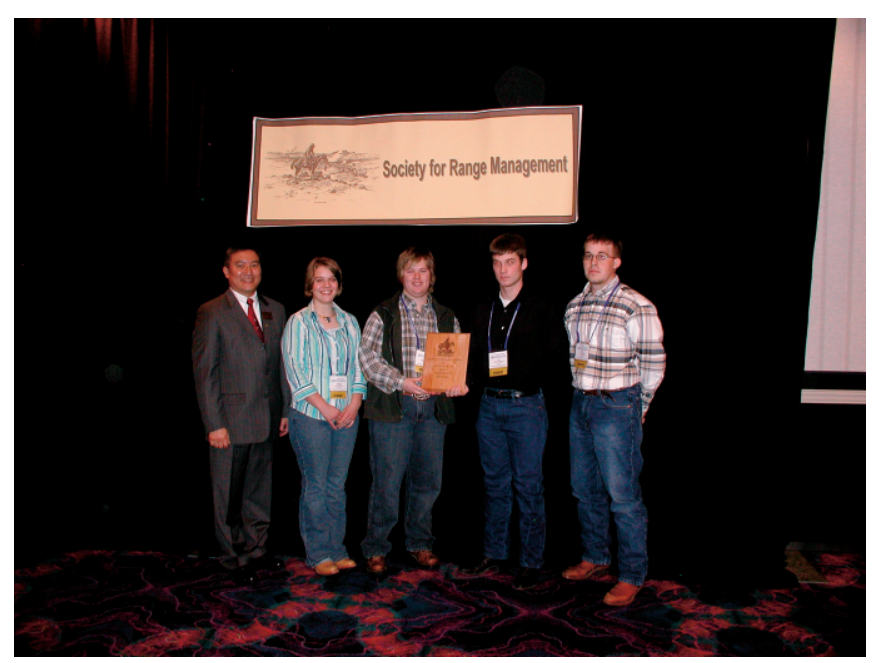

Rangelands Cup-Team: 3rd-Chadron State College: Kimmy Mott, Jenny Walker, Dan Ruterbories, Craig Eddie. 


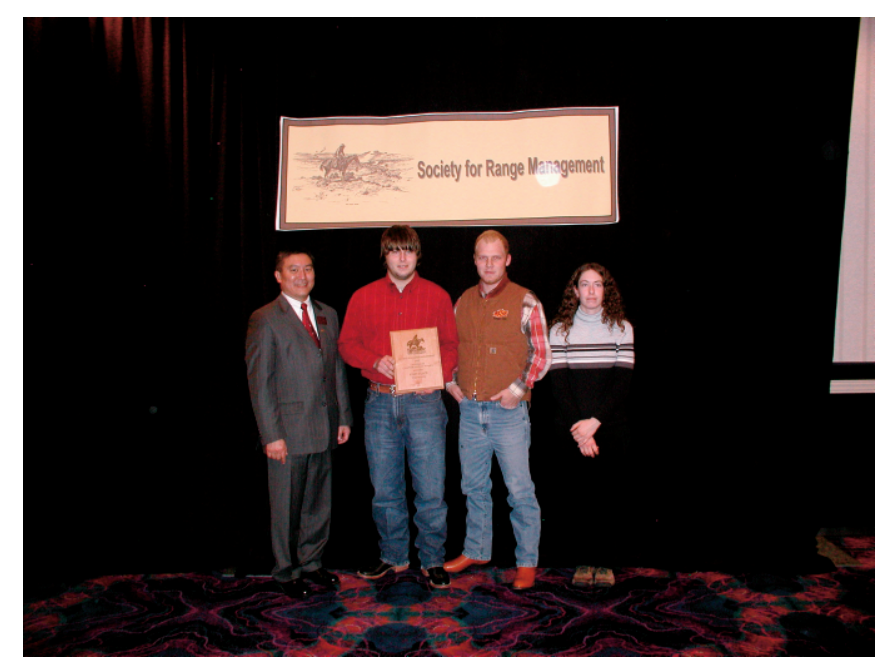

University Student Display: 1st-Oklahoma State University: Derek Matz, Lauren Wilkerson, Rusty Norrie.

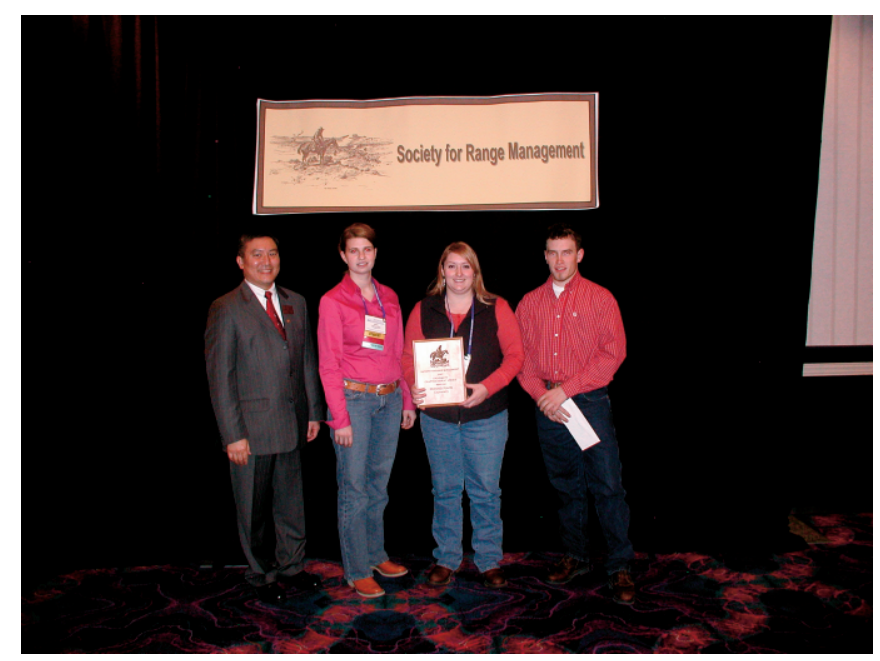

University Student Display: 2nd-Oregon State University: James Jaberg, Sheena Miltenberger, Jimmie Hayes.

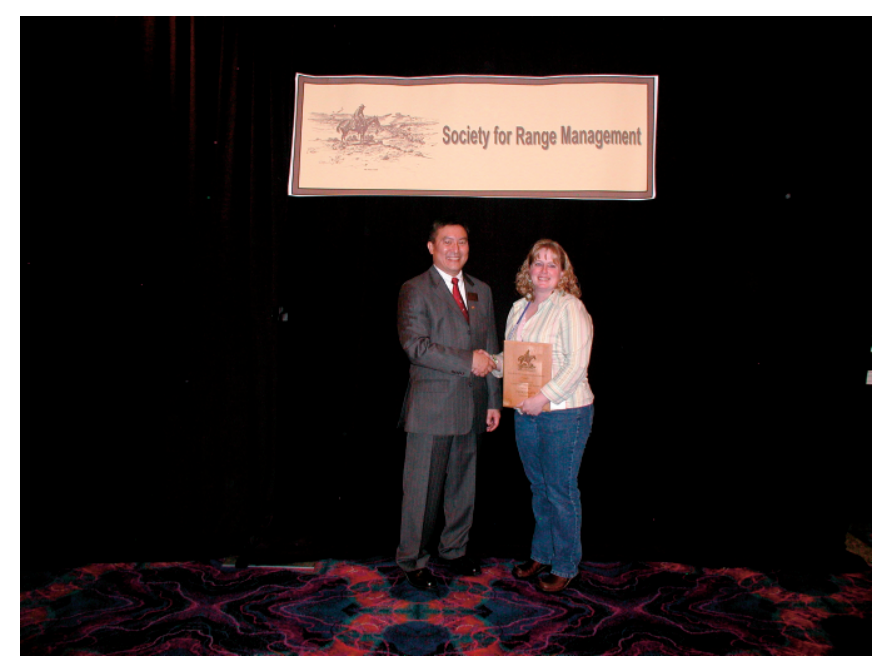

University Student Display: 3rd-University of Nevada, Reno: Michelle Burrows.

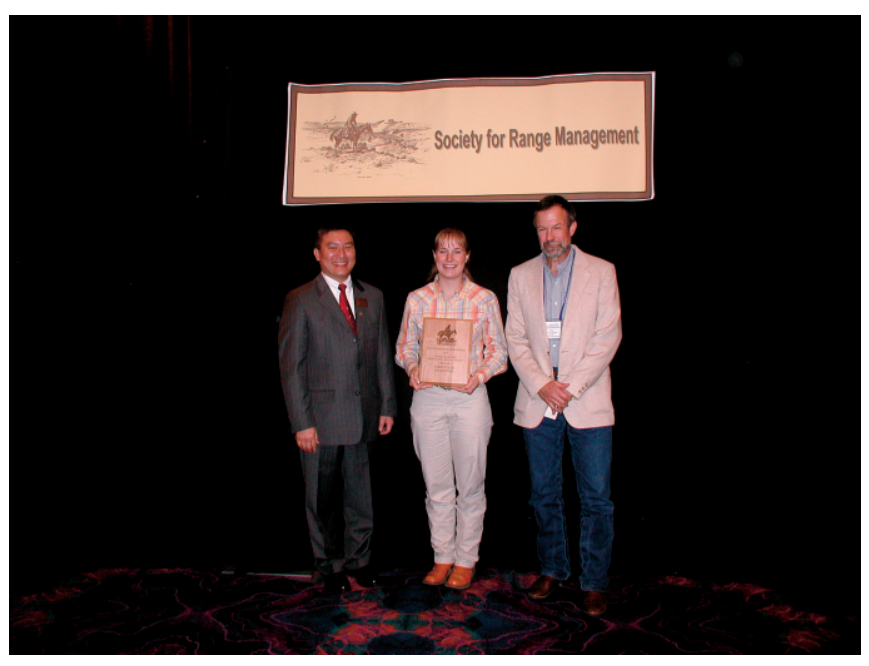

Undergraduate Public Speaking: 1st-Rives White, University of Wyoming with Tony Svejcar, USDA Agricultural Research Service.

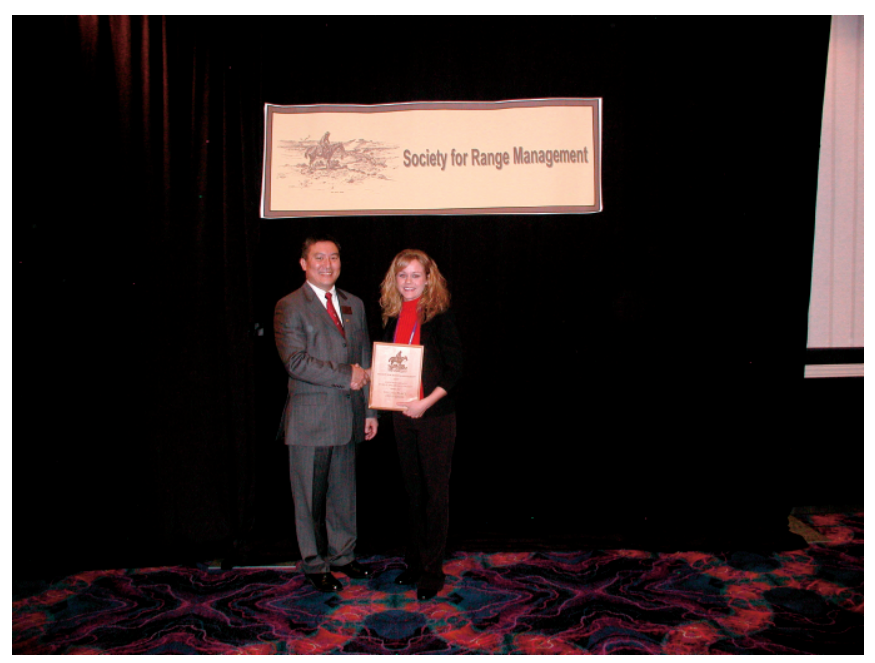

Undergraduate Public Speaking: 2nd-Courtney Ratheal, Texas A\&M University.

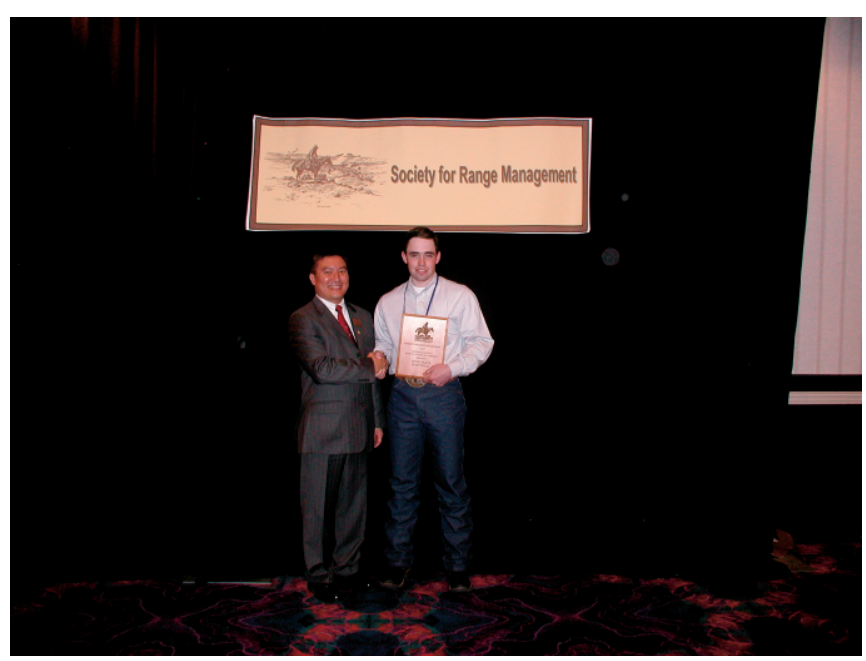

Undergraduate Public Speaking: 3rd-Mae Peterson, University of Wyoming 
High School Youth Forum (photos with papers in this issue)

1st-Colter Brown

Huntley Project High School-

Northern Great Plains Section

2nd-Bradley A. Fuller Clayton High School-New

Mexico Section

3rd-Melissa Barth

Foliett High School-Oklahoma Section

Belgrade High SchoolInternational Mountain Section

5th-Matthew Pfeifer Calhaun High School-Texas Section

\section{Graduate Student Competition}

(No photos.)

\section{Oral Paper Competition}

$P h D$

1st-Adena Rissman (University of California - Berkeley)

2nd (tie)-Ryan Limb (Oklahoma State University)

2nd (tie)-Stephen Winter (Oklahoma State University)

MS

1st-Kim Goodwin (Montana State University)

2nd-Corie Lund (North Dakota State University)

Poster Competition

$P h D$

1st-Erin Goergen (University of Nevada, Reno)

2nd-Matt Bahm (South Dakota State University)

MS

1st-Rae Haddow (University of Alberta)

2nd-Katie Benzel (Montana State University) 